\title{
6

\section{Effect of Aspiration Therapy on Obesity-Related Comorbidities: Systematic Review and Meta-Analysis}

\author{
Pichamol Jirapinyo', Diogo T. H. de Moura', Laura C. Horton ${ }^{2}$ and Christopher C. Thompson' \\ ${ }^{1}$ Division of Gastroenterology, Hepatology and Endoscopy, Brigham and Women's Hospital, Boston, MA, ${ }^{2}$ Department of Internal Medicine, \\ Brigham and Women's Hospital, Boston, MA, USA
}

Background/Aims: Aspiration therapy (AT) involves endoscopic placement of a gastrostomy tube with an external device that allows patients to drain $30 \%$ of ingested calories after meals. Its efficacy for inducing weight loss has been shown. This study aimed to assess the effect of AT on obesity-related comorbidities.

Methods: A meta-analysis of studies that assessed AT outcomes was conducted through December 2018. Primary outcomes were changes in comorbidities at 1 year following AT. Secondary outcomes were the amount of weight loss at up to 4 years and pooled serious adverse events (SAEs).

Results: Five studies with 590 patients were included. At 1 year, there were improvements in metabolic conditions: mean difference (MD) in systolic blood pressure: -7.8 (-10.7 - -4.9) mm Hg; MD in diastolic blood pressure: -5.1 (-7.0 - 3.2) mm Hg; MD in triglycerides: -15.8 (-24.0 - -7.6) mg/dL; MD in high-density lipoprotein: 3.6 (0.7-6.6) mg/dL; MD in hemoglobin Alc (HbA1c): -1.3 (-1.8 - -0.8) \%; MD in aspartate transaminase: $-2.7(-4.1--1.3) \mathrm{U} / \mathrm{L} ; \mathrm{MD}$ in alanine transaminase: $-7.5(-9.8--5.2) \mathrm{U} / \mathrm{L}$. At $1(n=218), 2(n=125), 3(n=46)$, and $4(n=27)$ years, the patients experienced $17.8 \%, 18.3 \%, 19.1 \%$, and $18.6 \%$ total weight loss (TWL), corresponding to $46.3 \%, 46.2 \%, 48.0 \%$, and $48.7 \%$ excess weight loss (EWL) ( $p<0.0001$ for all). Subgroup analysis of 2 randomized controlled trials $(n=225)$ showed that AT patients lost more weight than did controls by $11.6(6.5-16.7) \% \mathrm{TWL}$ and $25.6(16.0-35.3) \% \mathrm{EWL}$ and experienced greater improvement in $\mathrm{HbA1c}$ and alanine transaminase by $1.3(0.8-1.8) \%$ and $9.0(3.9-14.0) \mathrm{U} / \mathrm{L}$. The pooled SAE rate was $4.1 \%$.

Conclusions: Obesity-related comorbidities significantly improved at 1 year following AT. Additionally, a subgroup of patients who continued to use AT appeared to experience significant weight loss that persisted up to at least 4 years. Clin Endosc 2020;53:686-697

Key Words: Aspiration therapy; AspireAssist; Bariatric endoscopy; Comorbidities; Obesity

\section{INTRODUCTION}

Endoscopic bariatric and metabolic therapies (EBMTs) have recently been developed as an alternative treatment option for obesity. Compared to lifestyle intervention and pharmaco-

Received: September 11, 2019 Revised: October 31, 2019

Accepted: November 5, 2019

Correspondence: Christopher C. Thompson

Division of Gastroenterology, Hepatology and Endoscopy, Brigham and Women's Hospital, 75 Francis Street, Boston, MA 02115, USA

Tel: +1-617-525-8266, Fax: +1-617-264-6342, E-mail: cthompson@hms.harvard. edu

ORCID: https://orcid.org/0000-0002-6105-5270

(c) This is an Open Access article distributed under the terms of the Creative Commons Attribution Non-Commercial License (http://creativecommons.org/ licenses/by-nc/3.0) which permits unrestricted non-commercial use, distribution, and reproduction in any medium, provided the original work is properly cited. therapy, patients who undergo EBMTs generally achieve more significant weight loss, while maintaining a lower risk profile than those who undergo bariatric surgery. Additionally, for some patients with class I and II obesity who do not meet the criteria for surgery and those with class III obesity who do not want to undergo surgery, EBMTs may represent a feasible option for the treatment of obesity and potentially other metabolic comorbidities. ${ }^{1-6}$

Aspiration therapy (AT) is one of the available EBMTs that utilizes a device called the AspireAssist (Aspire Bariatrics, King of Prussia, PA, USA). The device was approved by the Food and Drug Administration in 2016 for long-term use in conjunction with lifestyle therapy (LT) for people with body mass index (BMI) of $35-55 \mathrm{~kg} / \mathrm{m}^{27}$. Accordingly, in this review, AT refers to AT in conjunction with LT. The device 
consists of the A-tube, which is a $26 \mathrm{Fr}$ percutaneous endoscopic gastrostomy tube with a $15-\mathrm{cm}$ fenestrated intragastric drainage catheter, a skin port, which connects to the external end of the A-tube and is normally closed to prevent gastric leakage, and a detachable connector, which connects with the skin port to allow aspiration of gastric contents. Participants aspirate approximately $30 \%$ of ingested calories at 30 minutes after meals, in addition to undergoing LT. After 115 uses, which is equivalent to 5 to 6 weeks of therapy, the connector locks and can no longer be used. Patients are required to see a practitioner who will provide a new connector as well as reinforce the importance of LT. It has been estimated that less than $80 \%$ of the weight loss is due to the aspiration of calories. The remaining $20 \%$ or more of the weight loss is thought to be due to a reduction in food intake. This is likely attributable to multiple factors including (1) food particles having to be less than or equal to $5 \mathrm{~mm}$ to fit through the A-tube, which likely leads to significantly longer chewing of food and reduced calorie consumption as a result; (2) increased water consumption to allow liquid gastric contents to flow out of the A-tube, which likely increases the sense of satiety without additional calories; and (3) the visibility of the gastric aspirate. Patients report that less healthy food options have an unappealing appearance on aspiration leading to a reduction in the consumption of those foods. $^{8}$

To date, several studies have reported the effectiveness of AT at inducing clinically significant weight loss. However, the effect of AT on obesity-related comorbidities remains unclear. This systematic review and meta-analysis aims to evaluate the changes in obesity-related comorbidities following AT. Additionally, the long-term effect of AT on weight profiles and its pooled serious adverse events (SAEs) will be assessed.

\section{MATERIALS AND METHODS}

\section{Data sources and searches}

The search strategy, study eligibility criteria, selection process, data collection process, primary and secondary outcomes, and analyses were defined $a$ priori and are described below.

We searched 3 databases-MEDLINE, EMBASE, and Web of Science-from inception to December 31, 2018 without language or study design restrictions. Keywords included "AspireAssist" and "Aspiration Therapy". Specifically, the search strategies included (AspireAssist[tiab] OR "aspiration therapy") for MEDLINE, Aspireassist OR "Aspiration Therapy" for EMBASE, and (AspireAssist) or ("Aspiration Therapy") for Web of Science. Duplicates were removed. Three of the authors (PJ, DTHM and LCH) then independently reviewed the titles and abstracts produced by the search. Studies deemed potentially relevant were reviewed in full to determine eligibility. Disagreements regarding final study inclusion were resolved by discussion with the senior author (CCT).

\section{Study selection and outcomes}

\section{Study design and population}

Randomized controlled trials (RCTs), observational studies, and case series that were peer reviewed and published as full text articles or presented as conference abstracts were included. Reviews, editorials, case-control studies, case reports, and studies using non-human subjects were excluded. If more than one study from the same research group was available, the authors were contacted to determine if the studies consisted of overlapping patient cohorts. If the patient cohorts overlapped, the study with a larger number of patients was selected to preserve the independence of the observations. If the study was a comparative study, only data from the AT group were included in the analysis. Studies were included if there were adult subjects (defined as aged $>18$ years) with class II obesity and above (BMI $\geq 35 \mathrm{~kg} / \mathrm{m}^{2}$ ) with at least 1 year of follow-up data. Corresponding authors were contacted for additional information if needed.

\section{Outcomes}

Primary outcomes were the changes in obesity-related comorbidities including hypertension, hyperlipidemia, type 2 diabetes mellitus (T2DM), and nonalcoholic fatty liver disease (NAFLD) at 1 year following A-tube placement. Specific surrogates for these comorbidities that were collected included systolic blood pressure (SBP), diastolic blood pressure (DBP), total cholesterol (CHOL), triglycerides (TG), low-density lipoprotein (LDL), high-density lipoprotein (HDL), hemoglobin Alc (HbAlc), aspartate transaminase (AST), and alanine transaminase (ALT).

Secondary outcomes were the changes in weight at 1, 2, 3, and 4 years following A-tube placement. Patients with less than 1 year of follow-up were excluded from this analysis. A subgroup analysis of only published studies that assessed the amount of weight loss in the AT group was also performed. Additionally, given the concern of possible increased adverse event (AE) rates following bariatric surgery in the elderly population, a subgroup analysis of studies that assessed the safety and efficacy of AT in patients aged $<55$ and $\geq 55$ years was performed. Furthermore, pooled SAEs were calculated and reported. Weight changes were reported using both percent total weight loss (\%TWL) and percent excess weight loss (\%EWL). 


\section{Data extraction and quality assessment}

Study characteristics, patient characteristics, and predefined primary and secondary outcomes were collected. The quality of observational studies and RCTs was evaluated using the Joanna Briggs Institute (JBI) Critical Appraisal Tools (non-comparative observational studies), Newcastle-Ottawa Quality Assessment Scale (NOS) (comparative observational studies), and JADAD score (RCTs). In this review, high quality was defined as meeting $\geq 50 \%$ of the JBI criteria, an NOS score of $\geq 6$, or a JADAD score of $\geq 3$. Two authors independently extracted data (PJ and DTHM) and assessed the quality of each of the studies (DTHM and LCH). Any disagreements were resolved by discussion with the senior author (CCT).

\section{Data synthesis and analysis}

Heterogeneity among studies was assessed using the $\chi^{2}$ test and $I^{2}$ statistic. Significant heterogeneity was defined as $p<0.05$ using the $\chi^{2}$ test or $I^{2}>50 \%$. A random-effects model was used to pool outcomes. Both intention-to-treat (ITT) and per-protocol (PP) data were pooled when available. Analyses were performed using Comprehensive Meta Analysis version 3.0 (Englewood, NJ, USA).

\section{RESULTS}

\section{Search results}

A total of 225 potential studies were identified, 24 of which were duplicates. After title and abstract review, 176 studies were excluded leaving 24 articles for full text review. Full text review yielded 5 articles that satisfied all criteria and were therefore included in the systematic review and meta-analysis (Fig. 1). ${ }^{8-12}$ All studies were graded as high quality (JBI score of $7, \mathrm{NOS}$ of 7 , and JADAD scores of 3,3 , and 3 ).

Of the 5 articles, 3 were published studies and 2 were conference abstracts (Table 1). ${ }^{8-12}$ The 5 articles detailed the results

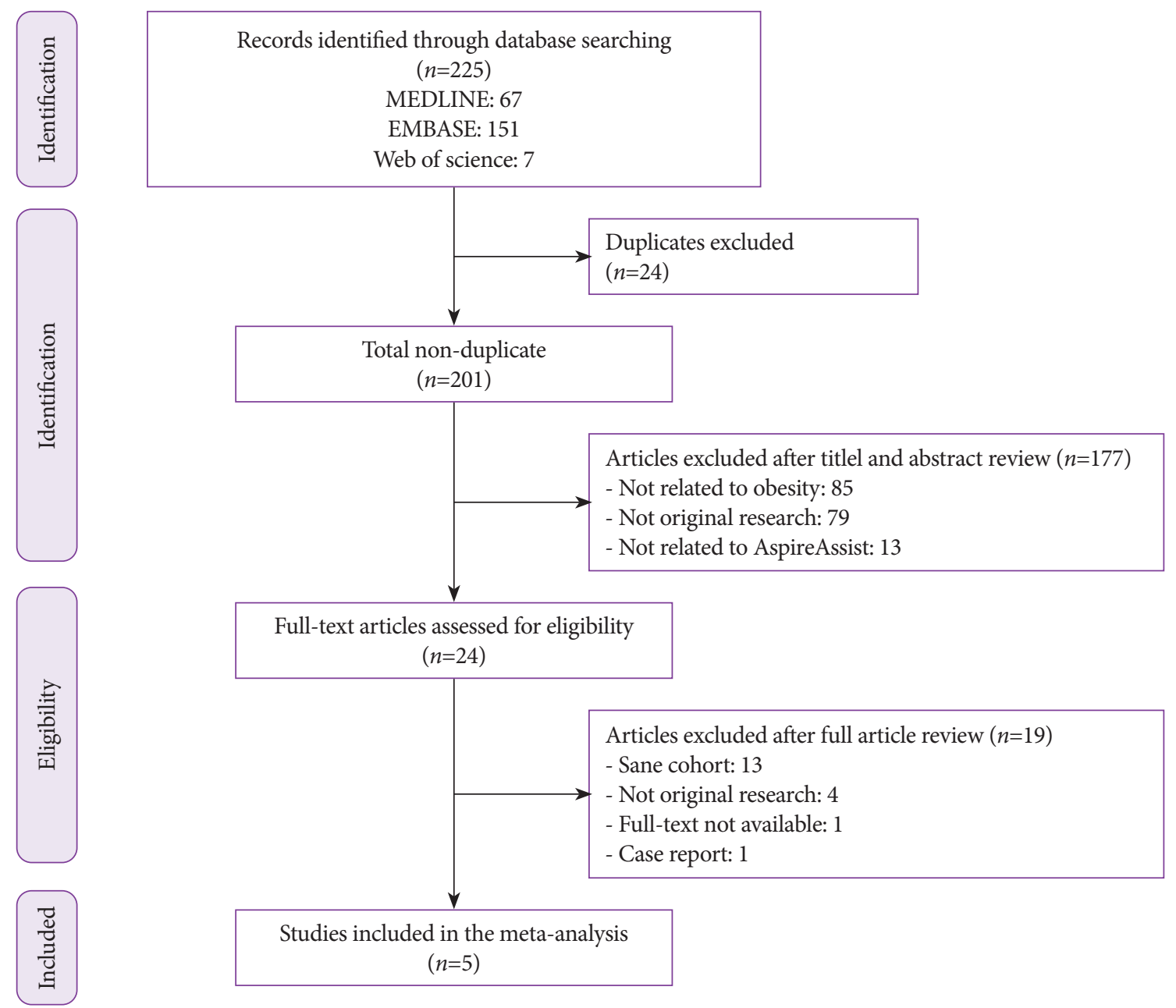

Fig. 1. Preferred Reporting Items for Systematic Reviews and Meta-Analyses (PRISMA) flow diagram. The search and selection process used for studies included in the meta-analysis. 
of 4 studies. Specifically, 1 abstract ${ }^{12}$ was a $2-4$-year follow-up report of a previously published study ${ }^{8}$ with a 1-year follow-up. Therefore, only the follow-up data (and not the 1-year data) from the abstract was used. Of the 4 non-overlapping studies, 2 were RCTs that compared AT to LT and 2 were observational studies, with 1 comparing AT to Roux-en-Y gastric bypass and the other being a non-comparative study. Only the AT arm of the 2 RCTs and the comparative observational study were included in the primary analysis of changes in obesity-related comorbidities, while both the AT and control arms of the RCTs were included in a subgroup analysis of the RCTs.

\section{Primary outcome}

\section{Obesity-related comorbidities}

All 4 included studies reported the effect of AT on obesity-related comorbidities, representing a total of 345 subjects who underwent AT. Mean age of the subjects ranged from 39 to 46 years. Mean BMI at the time of A-tube placement ranged from 42.4 to $43.5 \mathrm{~kg} / \mathrm{m}^{2}$. On average, the duration of A-tube placement ranged from 1 to 4 years.

ITT analysis: At 1 year, hypertension (SBP and DBP), hyperlipidemia (TG and HDL), T2DM (HbA1C), and NAFLD (AST and ALT) significantly improved. Specifically, SBP and DBP (4 studies with 218 patients) decreased by 7.8 ( $95 \%$ confidence interval, 4.9-10.7) $\mathrm{mm} \mathrm{Hg}$ and 5.1 (3.2-7.9) $\mathrm{mm} \mathrm{Hg}(p<0.0001$ for both), respectively. TG (4 studies with 209 patients) decreased by $15.8(7.6-24.0) \mathrm{mg} / \mathrm{dL}(p<0.0001)$, while HDL (2 studies with 93 patients) increased by $3.6(0.7-6.6) \mathrm{mg} / \mathrm{dL}$ ( $p=0.02$ ). HbA1c (3 studies with 23 patients) decreased by 1.3 $(0.8-1.8) \%(p<0.0001)$. Lastly, AST and ALT (2 studies with 93 patients) decreased by 2.7 (1.3-4.1) U/L and 7.5 (5.2-9.8) U/L ( $p<0.0001$ for both), respectively (Fig. 2, Table 2 ).

PP analysis: In addition to the above parameters, which were all significantly improved, LDL also significantly decreased at 1 year. Specifically, SBP and DBP (4 studies with 211 patients) decreased by 8.9 (5.3-12.4) $\mathrm{mm} \mathrm{Hg}$ and 5.0 (3.6-6.4) $\mathrm{mm} \mathrm{Hg}(p<0.0001$ for both), respectively. TG (4 studies with 200 patients) and LDL (2 studies with 91 patients) decreased by $20.4(11.7-29.1) \mathrm{mg} / \mathrm{dL}(p<0.0001)$ and $6.7(0.31-13.0) \mathrm{mg} / \mathrm{dL}(p=0.04)$, while HDL (2 studies with 92 patients) increased by $3.7(0.8-6.7) \mathrm{mg} / \mathrm{dL}(p=0.01)$. HbAlc (3 studies with 23 patients) decreased by $1.3(0.8-1.8) \%$ ( $p<0.0001)$. Lastly, AST and ALT (2 studies with 92 patients) decreased by 2.9 (1.4-4.3) U/L and 7.8 (5.4-10.1) U/L ( $p<0.0001$ for both), respectively (Table 2).

Table 1. Characteristics of Studies Included in the Meta-Analysis

\begin{tabular}{|c|c|c|c|c|c|c|c|c|}
\hline Study & Country & Study design & $\begin{array}{c}n \\
\text { (AT/Comparative } \\
\text { group) }\end{array}$ & $\begin{array}{l}\text { Age } \\
(y r)\end{array}$ & $\begin{array}{c}\text { Female } \\
(\%)\end{array}$ & $\begin{array}{l}\text { Duration } \\
\text { of AT (yr) }\end{array}$ & $\begin{array}{l}\text { Starting } \\
\text { BMI } \\
\left(\mathrm{kg} / \mathrm{m}^{2}\right)\end{array}$ & $\begin{array}{l}\text { Comorbidities } \\
\text { reported }\end{array}$ \\
\hline \multicolumn{9}{|l|}{ Published studies } \\
\hline $\begin{array}{l}\text { Sullivan et al. } \\
(2013)^{8}\end{array}$ & USA & $\begin{array}{c}\text { RCT } \\
(\mathrm{AT}+\mathrm{LT} \text { vs. } \mathrm{LT})\end{array}$ & $\begin{array}{c}18 \\
(11 / 7)\end{array}$ & $38.7 \pm 2.3$ & 93 & 2 & $42.6 \pm 1.4$ & $\begin{array}{l}\text { HTN, HLD, } \\
\text { NAFLD }\end{array}$ \\
\hline $\begin{array}{l}\text { Thompson et } \\
\text { al. }(2017)^{9}\end{array}$ & USA & $\begin{array}{c}\text { RCT } \\
(\mathrm{AT}+\mathrm{LT} \text { vs. } \mathrm{LT})\end{array}$ & $\begin{array}{c}207 \\
(137 / 70)\end{array}$ & $43.5 \pm 10.2$ & 84 & 1 & $42.2 \pm 5.1$ & $\begin{array}{l}\text { HTN, HLD, } \\
\text { T2DM, NAFLD }\end{array}$ \\
\hline $\begin{array}{l}\text { Nyström et } \\
\text { al. }(2018)^{10}\end{array}$ & $\begin{array}{l}\text { Czech, Italy, } \\
\text { Spain, } \\
\text { Netherlands, } \\
\text { Sweden }\end{array}$ & $\begin{array}{c}\text { Non-comparative } \\
\text { observational }\end{array}$ & 201 & $46.1 \pm 10.9$ & 75 & 4 & $43.6 \pm 7.2$ & $\begin{array}{l}\text { HTN, HLD, } \\
\text { T2DM }\end{array}$ \\
\hline \multicolumn{9}{|c|}{ Conference abstracts } \\
\hline $\begin{array}{l}\text { Wilson et al. } \\
(2018)^{11}\end{array}$ & Sweden & $\begin{array}{c}\text { Comparative } \\
\text { observational } \\
\text { (AT+LT vs. } \\
\text { RYGB+LT) }\end{array}$ & $\begin{array}{c}106 \\
(56 / 50)\end{array}$ & $41.0 \pm 11.0$ & 75 & 3 & $42.6 \pm 7.5$ & $\begin{array}{l}\text { HTN, HLD, } \\
\text { T2DM }\end{array}$ \\
\hline $\begin{array}{l}\text { Thompson et } \\
\text { al. }(2018)^{12 \mathrm{a})}\end{array}$ & USA & $\begin{array}{c}\text { Year 1: RCT } \\
\text { Years 2-4: } \\
\text { Non-comparative } \\
\text { observational }\end{array}$ & $\begin{array}{l}\text { Year 1: 207(137/70) } \\
\quad \text { Year 2-4: } 58\end{array}$ & $43.5 \pm 10.2$ & 84 & 4 & $42.2 \pm 5.1$ & N/A \\
\hline
\end{tabular}

Data presented as mean \pm standard deviation.

AT, aspiration therapy; BMI, body mass index; HLD, hyperlipidemia; HTN, hypertension; LT, lifestyle therapy; N/A, not available; NAFLD, nonalcoholic fatty liver disease; RCT, randomized controlled trial; RYGB, Roux-en-Y gastric bypass; T2DM, type 2 diabetes mellitus.

${ }^{\text {a) }}$ Follow-up study of Thompson 2017 study. Only data from years 2-4 were included in the analysis. 
Study

Sullivan et al. $(2013)^{8}$
Thompson et al. $(2017)^{9}$
Nyström et al. $(2018)^{10}$
Wilson et al. $(2018)^{11}$

(A)

$\underline{\text { Study }}$

Sullivan et al. $(2013)^{8}$
Thompson et al. $(2017)^{9}$
Nyström et al. $(2018)^{10}$
Wilson et al. $(2018)^{11}$

(B)

$\underline{\text { Study }}$

Sullivan et al. $(2013)^{8}$
Thompson et al. $(2017)^{9}$
Nyström et al. $(2018)^{10}$
Wilson et al. $(2018)^{11}$

(C)

$\underline{\text { Study }}$

Sullivan et al. $(2013)^{8}$
Thompson et al. $(2017)^{9}$
Nyström et al. $(2018)^{10}$
Wilson et al. $(2018)^{11}$

(D)

$\underline{\text { Study }}$

\begin{tabular}{|c|c|c|c|c|c|c|c|}
\hline & $\begin{array}{l}\text { Difference } \\
\text { in meams }\end{array}$ & $\begin{array}{l}\text { Standard } \\
\text { error }\end{array}$ & Variance & $\begin{array}{c}\text { Lower } \\
\text { limit }\end{array}$ & $\begin{array}{l}\text { Upper } \\
\text { limit }\end{array}$ & $\mathrm{Z}$-value & $p$-value \\
\hline llivan et al. $(2013)^{8}$ & -5.180 & 5.690 & 32.373 & -16.332 & 5.972 & -0.910 & 0.363 \\
\hline \multirow[t]{2}{*}{ Thompson et al. $(2017)^{9}$} & -6.000 & 3.760 & 14.134 & -13.369 & 1.369 & -1.596 & 0.111 \\
\hline & -5.751 & 3.317 & 9.839 & -11.899 & 0.397 & -1.833 & 0.067 \\
\hline
\end{tabular}

Statistics for each study

\begin{tabular}{|c|c|c|c|c|c|c|}
\hline $\begin{array}{l}\text { Difference } \\
\text { in meams }\end{array}$ & $\begin{array}{l}\text { Standard } \\
\text { error }\end{array}$ & Variance & $\begin{array}{l}\text { Lower } \\
\text { limit }\end{array}$ & $\begin{array}{l}\text { Upper } \\
\text { limit }\end{array}$ & $\mathrm{Z}$-value & $p$-value \\
\hline-12.000 & 3.784 & 14.319 & -19.417 & -4.583 & -3.171 & 0.002 \\
\hline-5.690 & 1.571 & 2.469 & -8.769 & -2.611 & -3.621 & 0.000 \\
\hline-10.300 & 2.118 & 4.484 & -14.450 & -6.150 & -4.864 & 0.000 \\
\hline-6.000 & 2.646 & 7.000 & -11.715 & -0.812 & -2.268 & 0.023 \\
\hline-7.490 & 1.091 & 1.189 & -9.627 & -5.352 & -6.868 & 0.000 \\
\hline
\end{tabular}

$\underline{\text { Statistics for each study }}$

Difference Standard

in meams error

$-7.200 \quad 2.210$

$-3.020 \quad 1.046$

$-5.800 \quad 1.269$

$-6.000 \quad 1.622$

Z-value $p$-value

$$
\begin{array}{lllll}
4.885 & -11.532 & -2.868 & -3.258 & 0.001
\end{array}
$$

$\begin{array}{lllll}1.095 & -5.070 & -0.970 & -2.887 & 0.004\end{array}$

$\begin{array}{lllll}1.611 & -8.288 & -3.312 & -4.570 & 0.000\end{array}$

$\begin{array}{lllll}2.632 & -9.180 & -2.820 & -3.698 & 0.000\end{array}$

$\begin{array}{lllll}0.472 & -6.119 & -3.426 & -6.948 & 0.000\end{array}$
$-4.773 \quad 0.687$

\section{Statistics for each study}

Difference Standard

in meams error

$-2.550 \quad 6.389$

$-6.000 \quad 4.201$

$13.200 \quad 5.082$

$23.000 \quad 5.528$

$6.842 \quad 6.971$

\section{Lower Upper}

limit limit

$\begin{array}{lll}40.814 & -15.071 & 9.971\end{array}$

$\begin{array}{lll}17.646 & -14.233 & 2.233\end{array}$

$\begin{array}{lll}25.824 & 3.240 & 23.160\end{array}$

$30.563 \quad 12.165 \quad 33.835$

$\begin{array}{lll}48.595 & 6.821 \quad 20.505\end{array}$
Z-value

$-0.399$

$-1.428$

2.598

4.160

0.982 $p$-value

0.690

0.153

0.009

0.000

0.326
Statistics for each study

Difference Standard

in meams error

$-10.910 \quad 14.857$

$-24.000 \quad 8.471$

$-16.900 \quad 7.242$

$-10.000 \quad 7.206$

$-15.825 \quad 4.196$ $\begin{array}{ccc} & \text { Lower } & \text { Upper } \\ \text { Variance } & \text { limit } & \text { limit }\end{array}$

$\begin{array}{lll}71.756 & -40.603 & -7.397\end{array}$

$\begin{array}{lll}52.450 & -31.095 & -2.705\end{array}$

$51.928-24.124-4.124$

$\begin{array}{lll}17.609 & -24.049 & -7.600\end{array}$
$220.736-40.030 \quad 18.210$

Z-value

p-value

$-0.734 \quad 0.463$
-2.833

$\begin{array}{ll}-2.833 & 0.005\end{array}$

$-2.334 \quad 0.020$

$-1.388 \quad 0.165$

$-3.771 \quad 0.000$

(E)

Fig. 2. Forest plots of the effects of aspiration therapy on obesity-related comorbidities at 1 year (intention-to-treat analysis). (A) Systolic blood pressure (mm Hg), (B) diastolic blood pressure $(\mathrm{mm} \mathrm{Hg}),(\mathrm{C})$ total cholesterol $(\mathrm{mg} / \mathrm{dL}),(\mathrm{D})$ triglycerides $(\mathrm{mg} / \mathrm{dL}),(\mathrm{E})$ low-density lipoprotein $(\mathrm{mg} / \mathrm{dL}),(\mathrm{F})$ high-density lipoprotein $(\mathrm{mg} / \mathrm{dL}),(\mathrm{G})$ hemoglobin $\mathrm{A} 1 \mathrm{C}(\%),(\mathrm{H})$ aspartate transaminase $(\mathrm{U} / \mathrm{L}),(\mathrm{I})$ alanine transaminase $(\mathrm{U} / \mathrm{L})$. $\mathrm{Cl}$, confidence interval. 
Study

$\underline{\text { Study }}$

\begin{tabular}{|c|c|c|c|c|c|c|c|}
\hline & $\begin{array}{l}\text { Difference } \\
\text { in meams }\end{array}$ & $\begin{array}{l}\text { Standard } \\
\text { error }\end{array}$ & Variance & $\begin{array}{l}\text { Lower } \\
\text { limit }\end{array}$ & $\begin{array}{l}\text { Upper } \\
\text { limit }\end{array}$ & Z-value & $p$-value \\
\hline Sullivan et al. $(2013)^{8}$ & 1.370 & 4.008 & 16.063 & -6.485 & 9.225 & 0.342 & 0.732 \\
\hline \multirow[t]{2}{*}{ Thompson et al. (2017) } & 4.000 & 1.604 & 2.573 & 0.856 & 7.144 & 2.494 & 0.013 \\
\hline & 3.637 & 1.489 & 2.218 & 0.718 & 6.556 & 2.442 & 0.015 \\
\hline
\end{tabular}

$\underline{\text { Study }}$

\begin{tabular}{lccccccc}
\hline & \multicolumn{2}{c}{$\begin{array}{c}\text { Difference Standard } \\
\text { in meams }\end{array}$} & $\begin{array}{c}\text { error } \\
\text { Variance }\end{array}$ & $\begin{array}{c}\text { Lower } \\
\text { limit }\end{array}$ & $\begin{array}{c}\text { Upper } \\
\text { limit }\end{array}$ & Z-value & $p$-value \\
Sullivan et al. $(2013)^{8}$ & -1.600 & 0.397 & 0.158 & -2.378 & -0.822 & -4.032 & 0.000 \\
Thompson et al. $(2017)^{9}$ & -0.940 & 0.406 & 0.165 & -1.736 & -0.144 & -2.314 & 0.021 \\
${\text { Wilson et al. }(2018)^{11}}$ & -1.200 & 0.682 & 0.465 & -2.537 & 0.137 & -1.760 & 0.078 \\
& -1.266 & 0.262 & 0.069 & -1.780 & -0.753 & -4.831 & 0.000
\end{tabular}

(G)

Study

Sullivan et al. $(2013)^{8}$
Thompson et al. $(2017)^{9}$
H

Study

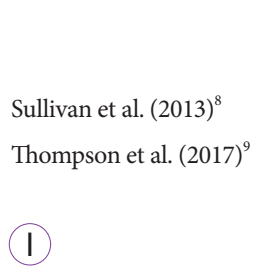

\begin{tabular}{ccccc} 
& & \multicolumn{3}{c}{ Statistics for each study } \\
\cline { 3 - 5 } $\begin{array}{c}\text { Difference } \\
\text { in meams }\end{array}$ & error & Variance & Lower & Upper \\
-7.100 & 2.139 & 4.574 & -11.292 & -2.908 \\
-7.630 & 1.386 & 1.921 & -10.347 & -4.913 \\
-7.473 & 1.163 & 1.353 & -9.753 & -5.194
\end{tabular}

Fig. 2. Continued.

\section{Secondary outcomes}

\section{Weight loss}

The 4 studies reported follow-up weight ranging from 2 to 4 years following the initiation of AT. All studies reported at least 2 years of follow-up, with 3 studies reporting a 3-year-follow up and 2 studies reporting a 4-year-follow-up. Fig. 3. shows a diagram of patient involvement and follow-up in the 2 studies with 4 -year follow-ups. Specifically, given that the patients could have chosen to withdraw from these 2 observational studies, i.e., have the A-tube removed, at any time, the number of patients who chose to withdraw between years $1-2,2-3$, and $3-4$ was 33,30 , and 9 , respectively. Of the patients who withdrew after the first year, $61.1 \%$ had achieved at least $10 \%$ TWL at the time of A-tube removal.
Difference in means and 95\% CI

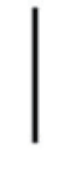

$-10.00$

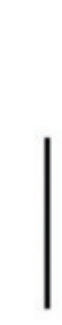

$-4.00$

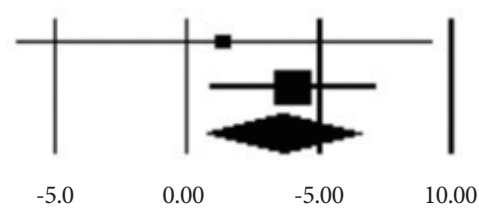

Difference in means and 95\% CI

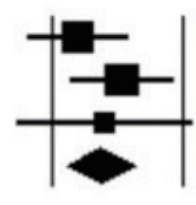

$-2.00$

0.00

2.00

4.00

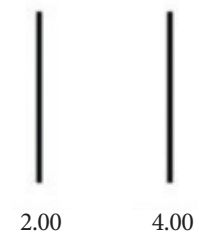

Z-value $p$-value

$-1.717 \quad 0.086$

$-3.298 \quad 0.001$

$-3.714 \quad 0.000$

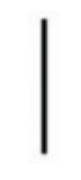

$-8.00$

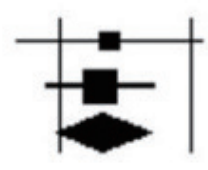

$-4.00$
Difference in means and 95\% CI

$\underline{\text { Difference in means and 95\% CI }}$

Z-value $p$-value

$\begin{array}{ll}-3.320 \quad 0.001 \\ -5.505 & 0.000\end{array}$

$\begin{array}{ll}-5.505 & 0.000\end{array}$

$-6.425 \quad 0.000$
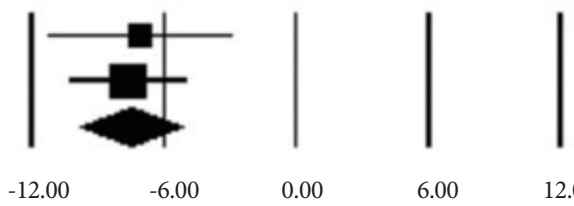

$-12.00$

$-6.00$

6.00

12.00
Per PP analysis, at 1 year (4 studies with 296 patients), the subjects experienced 17.8 (15.0-20.7) \%TWL $(p<0.0001)$, which corresponded to 46.3 (38.8-53.8) \%EWL ( $p<0.0001)$. At 2 years ( 4 studies with 174 patients), the subjects experienced 18.3 (15.7-20.9) \%TWL $(p<0.0001)$, which corresponded to 46.2 (42.1-50.4) \%EWL $(p<0.0001)$. At 3 years (3 studies with 88 patients), the subjects experienced 18.6 (16.4-20.8) \%TWL $(p<0.0001)$, which corresponded to $47.2(41.3-53.0) \% \mathrm{EWL}$ $(p<0.0001)$. At 4 years ( 2 studies with 27 patients), the subjects experienced 18.6 (13.9-23.4) \%TWL $(p<0.0001)$, which corresponded to 48.7 (35.7-61.7) \%EWL $(p<0.0001)$ (Table 3).

\section{Serious adverse events}

All 4 studies with a total of 345 AT participants reported SAEs, with a pooled SAE rate of $4.1 \%(14 / 345)$. These includ- 
Table 2. Summary of Meta-Analyses of Changes in Obesity-Related Comorbidities Compared to Baseline at 1 Year Following Initiation of Aspiration Therapy

\begin{tabular}{|c|c|c|c|c|}
\hline Comorbidities & $\begin{array}{l}\text { No. of studies } \\
\text { (No. of subjects) }\end{array}$ & $\begin{array}{c}\text { Mean difference } \\
(95 \% \mathrm{CI})\end{array}$ & $p$-value & $\begin{array}{c}I^{2} \\
(p \text {-value })\end{array}$ \\
\hline \multicolumn{5}{|l|}{ Intention-to-treat analysis } \\
\hline \multicolumn{5}{|l|}{ Hypertension } \\
\hline $\mathrm{SBP}(\mathrm{mm} \mathrm{Hg})$ & $4(218)$ & $-7.5(-9.6,-5.4)$ & $<0.0001$ & $37.6(0.19)$ \\
\hline $\mathrm{DBP}(\mathrm{mm} \mathrm{Hg})$ & $4(218)$ & $-4.8(-6.1,-3.4)$ & $<0.0001$ & $42.8(0.16)$ \\
\hline \multicolumn{5}{|l|}{ Hyperlipidemia } \\
\hline Total cholesterol (mg/dL) & $4(210)$ & $6.8(-6.8,20.5)$ & 0.33 & $86.0(<0.0001)$ \\
\hline Triglyceride (mg/dL) & $4(209)$ & $-15.8(-24.0,-7.6)$ & $<0.0001$ & $<0.0001(0.63)$ \\
\hline $\mathrm{LDL}(\mathrm{mg} / \mathrm{dL})$ & $2(93)$ & $-5.8(-11.9,0.4)$ & 0.07 & $<0.0001(0.90)$ \\
\hline $\mathrm{HDL}(\mathrm{mg} / \mathrm{dL})$ & $2(93)$ & $3.6(0.7,6.6)$ & 0.02 & $<0.0001(0.54)$ \\
\hline \multicolumn{5}{|l|}{ Type 2 Diabetes } \\
\hline Hemoglobin A1c (\%) & $3(23)$ & $-1.3(-1.8,-0.8)$ & $<0.0001$ & $<0.0001(0.51)$ \\
\hline \multicolumn{5}{|l|}{ NAFLD } \\
\hline AST (U/L) & $2(93)$ & $-2.7(-4.1,-1.3)$ & $<0.0001$ & $<0.0001(0.87)$ \\
\hline $\operatorname{ALT}(\mathrm{U} / \mathrm{L})$ & $2(93)$ & $-7.5(-9.8,-5.2)$ & $<0.0001$ & $<0.0001(0.84)$ \\
\hline \multicolumn{5}{|l|}{ Per protocol analysis } \\
\hline \multicolumn{5}{|l|}{ Hypertension } \\
\hline $\mathrm{SBP}(\mathrm{mm} \mathrm{Hg})$ & $4(211)$ & $-8.9(-12.4,-5.3)$ & $<0.0001$ & $55.5(0.08)$ \\
\hline $\mathrm{DBP}(\mathrm{mm} \mathrm{Hg})$ & $4(211)$ & $-5.0(-6.4,-3.6)$ & $<0.0001$ & $60.7(0.05)$ \\
\hline \multicolumn{5}{|l|}{ Hyperlipidemia } \\
\hline Total cholesterol (mg/dL) & $4(202)$ & $8.4(-7.4,24.2)$ & 0.30 & $88.7(<0.0001)$ \\
\hline Triglyceride (mg/dL) & $4(200)$ & $-20.4(-29.1,-11.7)$ & $<0.0001$ & $<0.0001(0.58)$ \\
\hline $\mathrm{LDL}(\mathrm{mg} / \mathrm{dL})$ & $2(91)$ & $-6.7(-13.0,-0.31)$ & 0.04 & $<0.0001(0.86)$ \\
\hline HDL (mg/dL) & $2(92)$ & $3.7(0.8,6.7)$ & 0.01 & $<0.0001(0.59)$ \\
\hline \multicolumn{5}{|l|}{ Type 2 Diabetes } \\
\hline Hemoglobin A1c (\%) & $3(23)$ & $-1.3(-1.8,-0.8)$ & $<0.0001$ & $<0.0001(0.51)$ \\
\hline \multicolumn{5}{|l|}{ NAFLD } \\
\hline $\operatorname{AST}(\mathrm{U} / \mathrm{L})$ & $2(92)$ & $-2.9(-4.3,-1.4)$ & $<0.0001$ & $<0.0001(0.85)$ \\
\hline $\operatorname{ALT}(\mathrm{U} / \mathrm{L})$ & $2(92)$ & $-7.8(-10.1,-5.4)$ & $<0.0001$ & $<0.0001(0.86)$ \\
\hline
\end{tabular}

ALT, alanine transaminase; AST, aspartate transaminase; CI, confidence interval; DBP, diastolic blood pressure; HDL, high-density lipoprotein; LDL, low-density lipoprotein; NAFLD, nonalcoholic fatty liver disease; SBP, systolic blood pressure.

ed buried bumper (2.3\%), peritonitis treated with intravenous antibiotics $(0.6 \%)$, severe abdominal pain treated with pain medication $(0.6 \%)$, abdominal pain secondary to pre-pyloric ulcer $(0.3 \%)$, and product malfunction requiring A-tube replacement $(0.3 \%)$.

Two studies reported a rate of persistent fistula following A-tube removal. At 1, 2, 3, and 4 years, the rates of persistent fistula were $2.2 \%(1 / 45), 1.6 \%(1 / 63), 39.3 \%$ (11/28), and 33.3\% (3/9), respectively. All but 2 fistula were closed successfully with 1-3 sessions of non-surgical interventions including brushing with a cytology brush, argon plasma coagulation and proton pump inhibitors, and endoscopic clips. Two pa- tients required surgical intervention for closure of a persistent fistula, representing $1.4 \%$ of all removed A-tubes.

\section{Subgroup analyses}

\section{Subgroup analysis of published studies only}

Three published studies with a total of 295 patients who underwent AT were included in the subgroup analysis of published studies only. Per ITT analysis, at 1 year, hypertension (SBP and DBP), hyperlipidemia (TG and HDL), T2DM (HbA1C), and NAFLD (AST and ALT) significantly improved. Specifically, SBP and DBP (3 studies with 168 patients) 
decreased by 8.6 (95\% confidence interval, 4.7-12.4) $\mathrm{mm} \mathrm{Hg}$ and 4.9 (2.5-7.3) $\mathrm{mm} \mathrm{Hg}(p<0.0001$ for both), respectively. TG (3 studies with 163 patients) decreased by 18.8 (8.7-28.9) $\mathrm{mg} / \mathrm{dL}(p<0.0001)$, while HDL (2 studies with 93 patients) increased by $3.6(0.7-6.6) \mathrm{mg} / \mathrm{dL}(p=0.02)$. HbAlc ( 2 studies with 17 patients) decreased by $1.3(0.7-1.8) \%(p<0.0001)$. Lastly, AST and ALT (2 studies with 93 patients) decreased by 2.7 (1.3-4.1) U/L and 7.5 (5.2-9.8) U/L ( $p<0.0001$ for both), re-

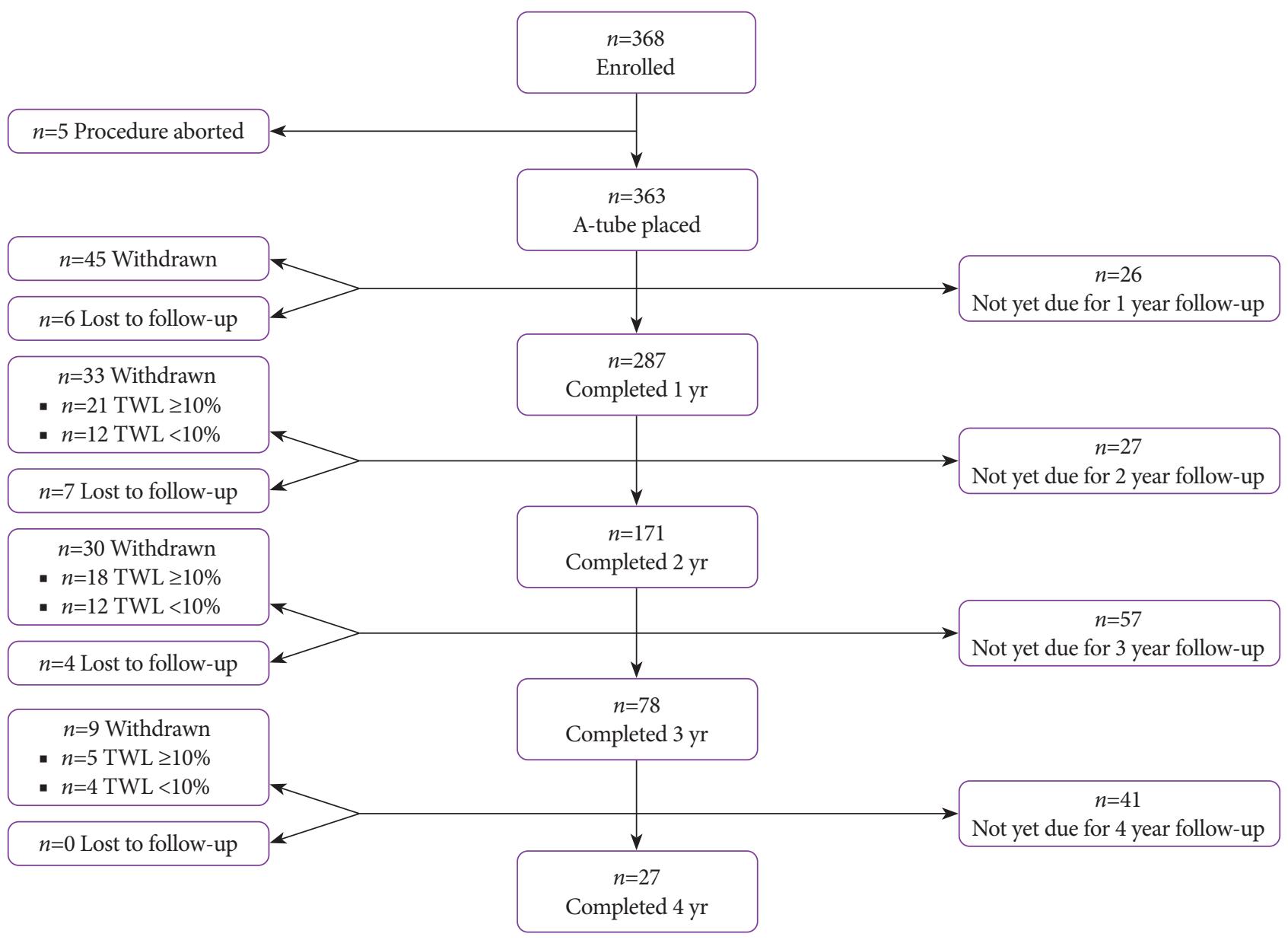

Fig. 3. Flow diagram of participant involvement in the 2 aspiration therapy studies with up to 4 -year follow-ups. TWL, total weight loss.

Table 3. Summary of Meta-Analyses of the Amount of Weight Loss Following Aspiration Therapy

\begin{tabular}{|c|c|c|c|c|}
\hline Duration of aspiration therapy & $\begin{array}{l}\text { No. of studies } \\
\text { (No. of subjects) }\end{array}$ & Amount of weight loss & $p$-value & $\begin{array}{c}I^{2} \\
(p \text {-value })\end{array}$ \\
\hline \multicolumn{5}{|l|}{ Total weight loss (\%) } \\
\hline $1 \mathrm{yr}$ & $4(296)$ & $17.8(15.0,20.7)$ & $<0.0001$ & $80.5(0.002)$ \\
\hline $2 \mathrm{yr}$ & $4(174)$ & $18.3(15.7,20.9)$ & $<0.0001$ & $55.6(0.08)$ \\
\hline $3 \mathrm{yr}$ & $2(46)$ & $19.1(14.5,23.7)$ & $<0.0001$ & $59.9(0.11)$ \\
\hline $4 \mathrm{yr}$ & $2(27)$ & $18.6(13.9,23.4)$ & $<0.0001$ & $<0.0001(0.84)$ \\
\hline \multicolumn{5}{|l|}{ Excess weight loss (\%) } \\
\hline $1 \mathrm{yr}$ & $4(296)$ & $46.3(38.8,53.8)$ & $<0.0001$ & $77.7(0.004)$ \\
\hline $2 \mathrm{yr}$ & $4(174)$ & $46.2(42.1,50.4)$ & $<0.0001$ & $1.0(0.39)$ \\
\hline $3 \mathrm{yr}$ & $2(46)$ & $48.0(40.0,56.0)$ & $<0.0001$ & $<0.0001(0.50)$ \\
\hline $4 \mathrm{yr}$ & $2(27)$ & $48.7(35.7,61.7)$ & $<0.0001$ & $<0.0001(0.92)$ \\
\hline
\end{tabular}


spectively. Per PP analysis, in addition to the above parameters that were all significantly improved, LDL also significantly decreased at 1 year. Specifically, SBP and DBP (3 studies with 167 patients) decreased by 9.3 (4.4-14.2) $\mathrm{mm} \mathrm{Hg}$ and 5.4 (2.4-8.3) $\mathrm{mm} \mathrm{Hg}$ ( $p<0.0001$ for both), respectively. TG (3 studies with 162 patients) and LDL (2 studies with 91 patients) decreased by $23.7(13.4-33.9) \mathrm{mg} / \mathrm{dL}(p<0.0001)$ and $6.7(0.31-13.0) \mathrm{mg} /$ $\mathrm{dL}(p=0.04)$, while HDL (2 studies with 92 patients) increased by $3.7(0.8-6.7) \mathrm{mg} / \mathrm{dL}(p=0.01)$. HbAlc (2 studies with 17 patients) decreased by $1.3(0.7-1.8) \%(p<0.0001)$. Lastly, AST and ALT ( 2 studies with 92 patients) decreased by 2.9 (1.4-4.3) U/L and 7.8 (5.4-10.1) U/L ( $p<0.0001$ for both), respectively.

\section{Subgroup analysis of randomized controlled trials}

Two RCTs with a total of 225 patients compared the effect of AT ( $n=148)$ to that of LT $(n=77)$. At 1 year, the patients in the AT arm experienced more weight loss than those in the LT arm by $11.6(6.5-16.7) \%$ TWL $(p<0.0001)$, which corresponded to 25.6 (16.0-35.3) \%EWL ( $p<0.0001)$. Additionally, the AT arm experienced greater improvement in $\mathrm{HbAlc}$ and ALT compared to the LT arm by $1.3(0.8-1.8) \%(p<0.0001$; $\left.I^{2}<0.0001\right)$ and $9.0(3.9-14.0) \mathrm{U} / \mathrm{L}\left(p<0.0001 ; I^{2}<0.0001\right)$, respectively. Other metabolic markers also improved to a greater extent in the $\mathrm{AT}$ arm than in the LT arm, although the differences were not statistically significant. Specifically, the AT arm experienced greater improvement in SBP, DBP, CHOL, TG, LDL, HDL, and AST compared to the LT arm by $3.5(-1.8-8.9) \mathrm{mm} \mathrm{Hg}\left(p=0.20 ; I^{2}<0.0001\right), 3.0(0.6-6.6)$ $\mathrm{mm} \mathrm{Hg}\left(p=0.10 ; I^{2}<0.0001\right), 2.0(-11.5-15.5) \mathrm{mg} / \mathrm{dL}$ ( $p=0.77$; $\left.I^{2}<0.0001\right), 15.2(-12.8-43.2) \mathrm{mg} / \mathrm{dL}\left(p=0.29 ; I^{2}<0.0001\right), 3.3$ $(-8.8-15.4) \mathrm{mg} / \mathrm{dL}\left(p=0.59 ; I^{2}<0.0001\right), 3.3(-2.2-8.8) \mathrm{mg} / \mathrm{dL}$ $\left(p=0.24 ; I^{2}<0.0001\right)$ and $1.8(-1.2-4.8) \mathrm{U} / \mathrm{L}\left(p=0.23 ; I^{2}=47.3\right)$, respectively.

\section{Subgroup analysis of different age groups}

Three studies with a total of 287 patients reported the amount of weight loss in younger patients (aged $<55$ years) $(n=232)$ and older patients (aged $\geq 55$ years) $(n=55)$. One study included only patients aged $<55$ years and was therefore not included in this sub-group analysis. ${ }^{7}$

At 1,2 , and 3 years, there was no statistically significant difference in the amount of weight loss between the 2 age subgroups. Specifically, at 1 year (3 studies with 287 patients), the older subjects experienced 17.9 (15.3-20.4) \%TWL, while the younger subjects experienced 19.2 (13.9-24.5) \%TWL ( $p=0.98$ ). At 2 years (3 studies with 170 patients), the older subjects experienced 19.7 (16.0-23.4) \%TWL, while the younger subjects experienced 17.0 (13.2-20.7) \%TWL ( $p=0.11)$. At 3 years ( 3 studies with 88 patients), the older subjects experienced
22.0 (18.3-25.8) \%TWL, while the younger subjects experienced 17.5 (15.1-19.9) \%TWL ( $p=0.30$ ).

At 4 years ( 2 studies with 27 patients), however, the older group experienced statistically significantly more weight loss compared to the younger group (31.5 [22.3-40.7] \%TWL in the older group vs. 16.9 [13.0-20.7] \%TWL in the younger group, $p=0.01$ ).

\section{DISCUSSION}

This systematic review and meta-analysis is the first to evaluate the effect of AT on metabolic comorbidities. Our study demonstrates that AT is associated with significant improvement in major metabolic outcomes including SBP, DBP, TG, HDL, LDL, HbAlc, AST, and ALT. Additionally, weight loss of approximately $17 \%$ to $19 \%$ of the baseline weight can be achieved and maintained up to at least 4 years following initiation of therapy, with an acceptable risk profile demonstrated by a $4.1 \%$ pooled SAE rate.

Obesity is a leading cause of preventable death in the U.S. that results in as much as $47 \%$ more life-years lost than tobacco use. ${ }^{13}$ Following obesity, other top modifiable risk factors in decreasing order include diabetes, tobacco use, hypertension, and hyperlipidemia, 3 of which (diabetes, hypertension, and hyperlipidemia) are direct and indirect adverse consequences of the increased body fat and adiposopathic dysfunction seen in patients with obesity. As a result, the goal of most obesity treatments is to improve metabolic outcomes, which will ultimately lead to a decrease in mortality risk.

Previous meta-analyses have shown the beneficial effects of other EBMTs including intragastric balloons (IGBs) and duodenal jejunal bypass liner (DJBL) on obesity-related comorbidities. Specifically, Popov et al. conducted a meta-analysis of 40 studies with 5,668 patients who underwent IGB placement. ${ }^{14}$ The study showed significant improvement in SBP by $9.1 \mathrm{~mm} \mathrm{Hg}$, DBP by $4.6 \mathrm{~mm} \mathrm{Hg}$, TG by $33.4 \mathrm{mg} / \mathrm{dL}$, HbAlc by $0.6 \%$, AST by $3 \mathrm{U} / \mathrm{L}$, and ALT by $9 \mathrm{U} / \mathrm{L}$ at the time of IGB removal at 6 months, when data from observational studies were pooled. ${ }^{14}$ More recently, Jirapinyo et al. conducted a meta-analysis on 14 studies with 412 patients with obesity and concomitant T2DM who underwent DJBL. ${ }^{15}$ At the time of DJBL removal at 12 months, patients experienced a significant improvement in T2DM with a decrease in HbAlc by $1.3 \%$, as well as improvement in insulin resistance and several gut hormones that control hunger, satiety, and glucose hemostasis. ${ }^{15}$

To the best of our knowledge, the present study is the first to elucidate the impact of AT on metabolic outcomes. Specifically, this study demonstrates that the chemical surrogates of the major metabolic conditions including hypertension, 
hyperlipidemia, T2DM, and NAFLD all significantly improve following AT. In contrast to prior studies on AT that focused primarily on its effect on weight loss, our study aimed to evaluate the effect of AT on metabolic comorbidities as the primary outcome. Additional data were obtained from every available study, allowing this meta-analysis to be sufficiently powered to detect changes in comorbidities, in contrast to prior studies that showed variable improvement in metabolic outcomes.

This improvement in metabolic functions is likely related to the amount and type of weight loss following AT. Specifically, a prospective study on 40 volunteers demonstrated that subjects who experienced $5 \%, 11 \%$, and $16 \%$ TWL had preferentially and disproportionately lost more intra-abdominal fat $(9 \%, 23 \%$, and $30 \%$, respectively) and intra-hepatic fat $(13 \%$, $52 \%$, and $65 \%$, respectively), which likely explained the stepwise metabolic benefits of weight loss at different levels. ${ }^{16} \mathrm{Ad}-$ ditionally, different tissues responded to different degrees of weight loss. While 5\% TWL significantly decreased glucose, insulin, TG, ALT, and leptin, only after 16\% TWL did plasma free fatty acid, C-reactive protein, and adiponectin improve. ${ }^{16}$ This evidence likely explains the findings that 1 ) at a TWL of $2 \%-5 \%$, there was an improvement in HbAlc, SBP, and TG; 2$)$ at a TWL of 5\%-10\%, there was an improvement in DBP and HDL; and 3) at a TWL of at least 10\%, there was an improvement in non-alcoholic steatohepatitis histologic features. ${ }^{17-19}$ In this study, AT was associated with $17.8 \%$ TWL at 1 year, which was above the $16 \%$ threshold for improvement in both metabolic/cardiovascular risk factors and inflammatory markers and likely explains the improvement in the chemical surrogates of all major obesity-related comorbidities shown in the study.

A challenge of most obesity treatments is long-term weight loss maintenance. While several definitions have been proposed to define successful weight loss maintenance, it is generally considered to be a sustained weight loss of 5\% to $10 \%$ of baseline weight in at least 1 year as recommended by the 2013 AHA/ACC/TOS Guideline for the Management of Overweight and Obesity in Adults. ${ }^{20,21}$ In our study, at 4 years following the initiation of AT, patients maintained their significant weight loss of $18.6 \%$ of their baseline weight, meeting the definition of successful weight loss maintenance. This suggests that the mechanisms of AT in combination with self-directed non-high-intensity LT $(<14$ lifestyle program sessions/ 6 months) were able to counteract physiological and cognitive adaptations favoring weight regain. In terms of the weight trends yielded by other treatment modalities, these results place AT closer to bariatric surgery than to lifestyle intervention and/or pharmacology. Specifically, a recent systematic review on the outcome of lifestyle intervention showed that weight loss reached its peak at 6 months after initiation of treatment. Without an active maintenance program, 50\% of patients had returned to their original weight at 5 years. ${ }^{22}$ In contrast, a recent study showed that patients who underwent Roux-en-Y gastric bypass experienced 35\% TWL at 2 years and were able to maintain their weight at $28 \%$ TWL and $26.9 \%$ TWL at 6 and 12 years, respectively. ${ }^{23}$

In our study, a subgroup analysis of different age groups showed no difference in weight outcomes following AT. In fact, at 4 years, the older patients experienced significantly greater weight loss (31.5\% TWL) compared to the younger group (16.9\% TWL). There have been controversies regarding the outcomes of bariatric and metabolic surgery in elderly patients, commonly defined as aged 55 years and older in most bariatric literature. Specifically, some studies showed that younger bariatric patients had better weight loss and comorbidity outcomes compared to the elderly group, ${ }^{24,25}$ although the data remained conflicted. ${ }^{26,27}$ Therefore, it has been suggested that bariatric surgical indications in elderly patients should be carefully considered. Given the minimal invasiveness of AT, acceptable safety profile of upper endoscopy, and favorable outcomes of AT in the elderly, ${ }^{28-30}$ it is possible that AT may have a broad application for those in this patient population who meet the BMI criteria.

Treatment with AT is accompanied by AEs, some of which are serious. In our study, the pooled SAE rate was $4.1 \%$, which is comparable to that of other EBMTs and within the risk threshold of $\leq 5 \%$ set by the ASGE/ASMBS Task Force on Endoscopic Bariatric Therapy. ${ }^{31,32}$ The most common SAE was buried bumper, which is a known complication of percutaneous endoscopic gastrostomy tubes that is thought be due to excessive tension on the internal bumper against the gastric wall. The buried bumpers were treated with removal of the A-tube, temporary replacement with a 20-Fr percutaneous endoscopic gastrostomy tube, followed by replacement with an A-tube. Persistent gastrocutaneous fistula following A-tube removal occurred, especially after 2 years of AT. All but 2 cases (1.4\% of all A-tubes removed) were successfully closed with endoscopic interventions.

This study has some limitations. First, the number of included studies was relatively small. To account for this, conference abstracts that met the a priori inclusion criteria were included in the analysis. Additionally, we were able to obtain additional data from all studies to make the analysis robust and sufficiently powered to detect beneficial changes in all obesity-related comorbidities. Another limitation is the fact that most of the published studies on AT were retrospective observational studies with a proportion of patients who were not yet due for follow-ups at the respective time points. Nevertheless, the primary outcome of this meta-analysis, which 
was the change in comorbidities at 1 year, was derived from a pool of almost 300 patients, making this a robust analysis. Another limitation is that each included study focused on weight loss as the primary outcome, with changes in comorbidities as a secondary outcome. These changes in metabolic parameters were reported for all subjects who underwent AT, including those with and without comorbidities at baseline. Additionally, the present study did not take into account the changes in anti-hypertensive, anti-hyperlipidemic, and anti-glycemic medications throughout the study period. Therefore, the changes in metabolic outcomes reported in this study were likely conservative and possibly underestimated the true effect of AT on obesity-related comorbidities.

In summary, this systematic review and meta-analysis suggests that AT is associated with significant improvement in hypertension, hyperlipidemia, T2DM, and NAFLD in patients with class II and III obesity. Moreover, substantial weight loss was experienced by this population, which persisted for at least 4 years in a subgroup of patients who continued to use the therapy appropriately. Given its simplicity and minimally invasive nature, AT may improve access to treatment in patients with obesity and concomitant metabolic comorbidities.

\section{Conflicts of Interest}

Christopher C. Thompson has conducted research for Aspire Bariatrics, USGI Medical, Spatz, and Apollo Endosurgery, has served as a consultant for Boston Scientific, Covidien, USGI Medical, Olympus, and Fractyl, holds stock and royalties for GI Windows and Endosim, and has served as an expert reviewer for GI Dynamics. The other authors have no financial conflicts of interest.

\section{Author Contributions}

Conceptualization: Christopher C. Thompson

Data curation: Pichamol Jirapinyo, Diogo T. H. de Moura, Laura C. Horton

Formal analysis: PJ, DTHM, LCH

Methodology: PJ, DTHM, LCH, CCT

Project administration: CCT

Resources: CCT

Software: CCT

Supervision: CCT

Validation: CCT

Visualization: CCT

Writing-original draft: PJ, DTHM, LCH

Writing-review\&editing: CCT

\section{ORCID}

Pichamol Jirapinyo: https://orcid.org/0000-0001-5273-6851

Diogo T. H. de Moura: https://orcid.org/0000-0002-7446-0355

Laura C. Horton: https://orcid.org/0000-0002-7098-3650

\section{REFERENCES}

1. Moura D, Oliveira J, De Moura EG, et al. Effectiveness of intragastric balloon for obesity: a systematic review and meta-analysis based on randomized control trials. Surg Obes Relat Dis 2016;12:420-429.

2. Jirapinyo P, Thompson CC. Endoscopic bariatric and metabolic therapies: surgical analogues and mechanisms of action. Clin Gastroenterol Hepatol 2017;15:619-630.

3. de Moura DTH, de Moura EGH, Thompson CC. Endoscopic sleeve gastroplasty: from whence we came and where we are going. World J Gastrointest Endosc 2019;11:322-328.

4. de Moura EGH, Ribeiro IB, Frazao MSV, et al. EUS-guided intragastric injection of botulinum toxin a in the preoperative treatment of super-obese patients: a randomized clinical trial. Obes Surg 2019;29:32-39.

5. Hourneaux De Moura DT, Thompson CC. Endoscopic management of weight regain following Roux-en-Y gastric bypass. Expert Rev Endocrinol Metab 2019;14:97-110.

6. de Moura DTH, de Moura EGH, Neto MG, Thompson CC. To the editor. Surg Obes Relat Dis 2019;15:155-157.

7. US Food and Drug Administration. Summary of safety and effectiveness data (SSED) [Internet]. Silver Spring (MD): FDA; c2016 [updated 2016 Jun 14; cited 2019 May 1]. Available from: https://www.accessdata. fda.gov/cdrh_docs/pdf15/p150024b.pdf.

8. Sullivan S, Stein R, Jonnalagadda S, Mullady D, Edmundowicz S. Aspiration therapy leads to weight loss in obese subjects: a pilot study. Gastroenterology 2013;145:1245-1252.e1-e5.

9. Thompson CC, Abu Dayyeh BK, Kushner R, et al. Percutaneous gastrostomy device for the treatment of class II and class III obesity: results of a randomized controlled trial. Am J Gastroenterol 2017;112:447-457.

10. Nyström M, Machytka E, Norén E, et al. Aspiration therapy as a tool to treat obesity: 1- to 4-year results in a 201-patient multi-center post-market European registry study. Obes Surg 2018;28:1860-1868.

11. Wilson EB, Noren E, Gruvaeus J, Paradis C. A comparative 100-participant 5-year study of aspiration therapy versus Roux-en-y gastric bypass: 2nd and 3rd year results. Surg Obes Relat Dis 2018;14(11 Suppl):S15.

12. Thompson CC, Dayyeh BKA, Kushnir V, et al. Aspiration therapy for the treatment of obesity: 2-4 year results of the PATHWAY multicenter randomized controlled trial. Surg Obes Relat Dis 2018;14(11 Suppl):S4-S5.

13. Cleveland Clinic. Obesity is top cause of preventable life-years lost, study shows [Internet]. Rockville (MD): Science Daily; c2017 [updated 2017 Apr 22; cited 2019 May 1]. Available from: https://www.sciencedaily.com/releases/2017/04/170422101614.htm.

14. Popov VB, Ou A, Schulman AR, Thompson CC. The impact of intragastric balloons on obesity-related co-morbidities: a systematic review and meta-analysis. Am J Gastroenterol 2017;112:429-439.

15. Jirapinyo P, Haas AV, Thompson CC. Effect of the duodenal-jejunal bypass liner on glycemic control in patients with type 2 diabetes with obesity: a meta-analysis with secondary analysis on weight loss and hormonal changes. Diabetes Care 2018;41:1106-1115.

16. Magkos F, Fraterrigo G, Yoshino J, et al. Effects of moderate and subsequent progressive weight loss on metabolic function and adipose tissue biology in humans with obesity. Cell Metab 2016;23:591-601.

17. Wing RR, Lang W, Wadden TA, et al. Benefits of modest weight loss in improving cardiovascular risk factors in overweight and obese individuals with type 2 diabetes. Diabetes Care 2011;34:1481-1486.

18. Lazo M, Solga SF, Horska A, et al. Effect of a 12-month intensive lifestyle intervention on hepatic steatosis in adults with type 2 diabetes. Diabetes Care 2010;33:2156-2163.

19. Promrat K, Kleiner DE, Niemeier HM, et al. Randomized controlled trial testing the effects of weight loss on nonalcoholic steatohepatitis. Hepatology 2010;51:121-129.

20. Rössner S. Defining success in obesity management. Int J Obes Relat Metab Disord 1997;21 Suppl 1:S2-S4.

21. Jensen MD, Ryan DH, Apovian CM, et al. 2013 AHA/ACC/TOS guideline for the management of overweight and obesity in adults: a report of the American College of Cardiology/American Heart Association Task Force on Practice Guidelines and The Obesity Society. Circulation 2014;129:S102-S138. 
22. Wing RR. Behavioral weight control. In: Wadden TA, Stunkard AJ, eds. Handbook of obesity treatment. New York (NY): Guilford Press; 2002. p. 301-316.

23. Adams TD, Davidson LE, Litwin SE, et al. Weight and metabolic outcomes 12 years after gastric bypass. N Engl J Med 2017;377:1143-1155.

24. Scozzari G, Passera R, Benvenga R, Toppino M, Morino M. Age as a long-term prognostic factor in bariatric surgery. Ann Surg 2012;256:724728; discussion 728-729.

25. Contreras JE, Santander C, Court I, Bravo J. Correlation between age and weight loss after bariatric surgery. Obes Surg 2013;23:1286-1289.

26. Lynch J, Belgaumkar A. Bariatric surgery is effective and safe in patients over 55: a systematic review and meta-analysis. Obes Surg 2012;22:15071516.

27. Giordano S, Victorzon M. Laparoscopic Roux-en-Y gastric bypass is effective and safe in over 55-year-old patients: a comparative analysis. World J Surg 2014;38:1121-1126.

28. Lee TC, Huang SP, Yang JY, et al. Age is not a discriminating factor for outcomes of therapeutic upper gastrointestinal endoscopy. Hepatogastroenterology 2007;54:1319-1322.

29. Lockhart SP, Schofield PM, Gribble RJ, Baron JH. Upper gastrointestinal endoscopy in the elderly. Br Med J (Clin Res Ed) 1985;290:283.

30. Travis AC, Pievsky D, Saltzman JR. Endoscopy in the elderly. Am J Gastroenterol 2012;107:1495-1501; quiz 1494, 1502.

31. ASGE/ASMBS Task Force on Endoscopic Bariatric Therapy, Ginsberg GG, Chand B, et al. A pathway to endoscopic bariatric therapies. Gastrointest Endosc 2011;74:943-953.

32. ASGE/ASMBS Task Force on Endoscopic Bariatric Therapy. A pathway to endoscopic bariatric therapies. Surg Obes Relat Dis 2011;7:672-682. 